\title{
Metronomic chemotherapy remodel cancer-associated fibroblasts to decrease chemoresistance of gastric cancer in nude mice
}

\author{
CHAO WANG $^{1 *}$, WENQI XI ${ }^{1 *}$, JINLING JIANG $^{1}$, JUN JI ${ }^{2}$, YINGYAN YU ${ }^{2}$, ZHENGGANG ZHU ${ }^{1,2}$ and JUN ZHANG ${ }^{1}$ \\ Departments of ${ }^{1}$ Oncology and ${ }^{2}$ Surgery, Ruijin Hospital, \\ Shanghai Jiaotong University School of Medicine, Shanghai 200025, P.R. China
}

Received August 19, 2016; Accepted September 13, 2017

DOI: $10.3892 / \mathrm{ol} .2017 .7197$

\begin{abstract}
The present study aimed to evaluate whether capecitabine or 5-fluorouracil (5-Fu) chemotherapy with the metronomic pattern may cause significant chemoresistance compared with the traditional pattern, and whether CAFs are involved in drug resistance. SGC-7901 cells were subcutaneously injected into the nude mice, and the mice were divided into five groups: The control group, intraperitoneally injected with normal saline; the 5-Fu conventional dose group [5-Fu maximum tolerated dose (MTD) group], intraperitoneally injected with $50 \mathrm{mg} / \mathrm{kg}$, twice per week for 2 weeks, with an 1-week discontinuation for 6 weeks; the capecitabine conventional dose group (capecitabine MTD group), intragastric $500 \mathrm{mg} / \mathrm{kg}$, twice per week for 2 weeks, with a 1-week discontinuation for 6 weeks; the 5-Fu metronomic group [5-Fu low-dose metronomic (LDM) group], intraperitoneally injected with $15 \mathrm{mg} / \mathrm{kg}$, twice a week for 6 weeks; and the capecitabine metronomic group (capecitabine LDM group), intragastric administration at $200 \mathrm{mg} / \mathrm{kg}$, twice a week for 6 weeks. The chemotherapy resistance markers [glutathione transferase $\mathrm{Pi}$ (GSTP) and multidrug resistance protein 1 (MDR1)] were detected by immunohistochemical staining (IHC), and the association of the expression of these markers with the chemotherapy administration patterns was analyzed. Vascular endothelial growth factor (VEGF) and the cancer-associated fibroblast (CAF) marker $\alpha$-smooth muscle actin were also examined by IHC to illustrate the possible mechanism of chemoresistance. The expression of GSTP and MDR1 in the MTD groups was significantly higher compared with those of the LDM groups $(\mathrm{P}<0.01)$. Furthermore, the number of CAFs
\end{abstract}

Correspondence to: Dr Jun Zhang, Department of Oncology, Ruijin Hospital, Shanghai Jiaotong University School of Medicine, 197 Ruijin Er Road, Shanghai 200025, P.R. China

E-mail: junzhang10977@sjtu.edu.cn

${ }^{*}$ Contributed equally

Key words: metronomic chemotherapy, 5-fluorouracil, capecitabine, chemoresistance, vascular endothelial growth factor, cancer-associated fibroblast and the level of VEGF in the MTD groups were significantly higher compared with those of the LDM groups $(\mathrm{P}<0.05)$. The low dose metronomic chemotherapy did not increase the risk of chemoresistance compared with the conventional dose traditional chemotherapy in terms of capecitabine or 5-Fu, the increasing amount of CAFs in the microenvironment of cancer cell following therapy may protect cell from capecitabine or 5-Fu via producing VEGF to increase vascularization.

\section{Introduction}

Gastric cancer (GC) is one of the leading causes of cancer-associated mortality worldwide, and the incidence rate of this disease is high, particularly in Eastern Asia (1). According to a study in 2012, there were an estimated 951,600 cases of newly diagnosed GC and 723,100 GC-associated mortalities (1), thus, the application of effective treatment for GC is urgent. Surgical resection with lymph node dissection is the cornerstone for treatment of GC, particularly for those in the early stage; however, the majority of patients are diagnosed at an advanced stage in Eastern Asia, and even a number of those receiving radical surgery have local and systemic recurrence (2). Several clinical trials have been performed to compare between surgery with adjuvant chemotherapy following curative D2 gastrectomy or neoadjuvant chemotherapy prior to D2 or more extended surgery, and surgery alone, including the ACTS-GC, CLASSIC, NSAS-GC, JCOG 0501 and PRODIGY studies (2-5). The results of those trials demonstrated that adjuvant chemotherapy with surgery may be more beneficial to patients compared with surgery alone, and the phase III trials of neoadjuvant chemotherapy (JCOG 0501 and PRODIGY) are still going on.

Metronomic chemotherapy, in last decade, has been gradually recognized and became an alternative to conventionally scheduled chemotherapy. The notion of 'high time for low dose' has replaced 'the higher the dose, the better' with the purpose of administering systemic therapy incessantly with minimal side effects (6). Metronomic chemotherapy not only disrupts the process of cell division, which inhibits the proliferation of cancer cell, but also eliminates endothelial cells involved in angiogenesis, termed an anti-angiogenetic effect (7). In our previous studies, capecitabine and 5-fluorouracil $(5-\mathrm{Fu})$ was able to express a marked anti-angiogenetic effect when administered at defined doses and schedules in mice xenografts of 
gastrointestinal cancer cell lines $(8,9)$. Besides tapering the tumor growth, the anti-angiogenetic activity of metronomic chemotherapy was able to overcome drug resistance (10). It is known that inherent and acquired resistance are one of the major hinders for chemotherapy (11). In recent years, studies on chemotherapy resistance have focused on the tumor microenvironment. Cancer-associated fibroblasts (CAFs), the dominant component of the tumor microenvironment, have been confirmed to modulate chemoresistance by secreting cytokines, including stromal cell-derived factor- $1 \alpha$, interleukin (IL)-6 and IL-17A (12-14). The present study aimed to evaluate whether capecitabine or 5-Fu chemotherapy with the metronomic pattern may cause significant chemoresistance compared with the traditional pattern, and whether CAFs are involved in the drug resistance.

\section{Materials and methods}

Cell lines and culture. Human GC cell line, SGC-7901, was obtained from Shanghai Institute of Digestive Surgery (Shanghai, China). These cells were maintained in Dulbecco's modified Eagle's medium (BasalMedia; Thermo Fisher Scientific, Inc., Waltham, MA, USA) supplemented with $10 \%$ fetal bovine serum (AusgeneX Pty Ltd., Gold Coast, Australia) at $37^{\circ} \mathrm{C}$ with $5 \% \mathrm{CO}_{2}$ and saturated humidity.

Establishment of GC xenografts and tissue collection. Male Balb/c nude mice $(\mathrm{n}=25)$, 4-6 weeks of age, with body weight of 15-20 g, were provided by the Research Center of Experimental Medicine, Shanghai Jiaotong University School of Medicine Affiliated Ruijin Hospital (Shanghai, China). Mice received humane care, and the study protocol was approved by the Animal Care and Use Committee and conducted in accordance with the Guide for the Care and Use Laboratory Animals of Ruijin Hospital, Shanghai Jiaotong University School of Medicine. Prior to performing the experiment, animals were placed in separate cages for 1 week to adapt to the new environment, which was under specific pathogen-free (SPF) conditions. The temperature was maintained at between 22 and $25^{\circ} \mathrm{C}$, with between 40 and $70 \%$ relative humidity, a 12-h light/12-h dark cycle and a light intensity of between 15 and 20 lux. Water in drinking bottles and pelleted food (Xietong-organism, Nanjing, China) were provided ad libitum. The SGC-7901 cell suspension was adjusted to a cell density of $1 \times 10^{7} / \mathrm{ml}$, and the nude mice were subcutaneously inoculated with a $100 \mu$ l suspension. Administration of the therapy was initiated when the subcutaneous nodules were $\sim 2 \mathrm{~mm}$ in diameter. The nude mice were randomly divided into the following groups: i) Control group, intraperitoneally injected with normal saline; ii) 5-Fu conventional dose group [5-Fu maximum tolerated dose (MTD) group], intraperitoneally injected with $50 \mathrm{mg} / \mathrm{kg}$, twice per week for 2 weeks, with a 1 week discontinuation for 6 weeks; iii) 5 -Fu metronomic group [5-Fu low-dose metronomic (LDM) group], intraperitoneally injected with $15 \mathrm{mg} / \mathrm{kg}$, twice a week for 6 weeks; iv) capecitabine (Roche Diagnostics, Shanghai, China) conventional dose (capecitabine MTD group), intragastric $500 \mathrm{mg} / \mathrm{kg}$, twice per week for 2 weeks, with a 1 week discontinuation for 6 weeks; and v) capecitabine metronomic group (capecitabine LDM group), intragastric administration at
$200 \mathrm{mg} / \mathrm{kg}$, twice a week for 6 weeks. Following drug administration, a Vernier caliper was used to measure the length (L) and short track $(\mathrm{W})$ of the tumor mass every 7 days in order to calculate the volume $(\mathrm{V})$ according to the following formula: $\mathrm{V}=(\mathrm{W}+\mathrm{L}) /(2 \times \mathrm{W} \times \mathrm{L} \times 0.5236)(9)$. Tumor size did not exceed $20 \mathrm{~mm}$ in any direction.

Immunohistochemical staining (IHC). The tumor was fixed with $10 \%$ formaldehyde for $24 \mathrm{~h}$ at room temperature. Following hematoxylin-eosin staining (30 min; room temperature) for tumor confirmation, immunohistochemical staining was performed on $4-\mu \mathrm{m}$ sections following the EnVision two-step procedure of DakoREAL ${ }^{\mathrm{TM}}$ EnVision $^{\mathrm{TM}}$ Detection system (Dako; Agilent Technologies GmbH, Waldbronn, Germany). The slides were incubated at $4^{\circ} \mathrm{C}$ overnight with primary antibodies for GSTP (dilution, 1:100; catalog no. GT202729; Dako; Agilent Technologies GmbH), MDR1 (dilution, 1:50; catalog no. BM0508; Wuhan Boster Biological Technology, Ltd. Wuhan, China), $\alpha$-smooth muscle actin ( $\alpha$-SMA; dilution, 1:50; catalog no. GM085129; Dako; Agilent Technologies GmbH), CD34 (dilution, 1:200; catalog no. SC-9095; Santa Cruz Biotechnology, Inc., Dallas, TX, USA) and vascular endothelial growth factor (VEGF; dilution, 1:50; catalog no. M727329; Dako; Agilent Technologies $\mathrm{GmbH})$. The horseradish peroxidase-labeled antibody to rabbit and mouse immunoglobulin were used as secondary antibodies (used as supplied; catalog no. K5007; Dako; Agilent Technologies $\mathrm{GmbH}$ ) incubated at $37^{\circ} \mathrm{C}$ for $30 \mathrm{~min}$. The slides were visualized by diaminobenzidine under a light microscope (BX51, Olympus Corporation, Tokyo, Japan; x200 magnification). The staining result criteria were as follows: A tumor with brownish-yellow granules was positive for antibody staining. Image-Pro Plus 6.0 (Media Cybernetics, Inc., Rockville, MD, USA) was used to measure the mean density of positive staining, which was the equivalent to the total integrated optical density/area.

Statistical analysis. SPSS software (version 13.0; SPSS, Inc., Chicago, IL, USA) was used for statistical analysis. Data are presented as the mean \pm standard deviation, and differences between the groups were compared using one-way analysis of variance with the Tukey's multiple comparison post hoc test. $\mathrm{P}<0.05$ was considered to indicate a statistically significant difference.

\section{Results}

Expression of chemoresistance markers is increased following chemotherapy. Our previous studies have confirmed that metronomic 5-Fu-based chemotherapy may perform an anti-angiogenetic role, which is associated with the antitumor effects of metronomic chemotherapy in vivo and in vitro. Compared with the conventional dose traditional chemotherapy, the antitumor effect of low dose metronomic chemotherapy is not inferior to the former $(8,9)$. In addition to the anti-angiogenetic effect, it was speculated that there are other factors that enhance the antitumor effect of low-dose groups. Thus, the expression of chemoresistance markers, including GSTP and MDR1, was examined in the two groups. The expression of GSTP and MDR1 in GC was determined 
by IHC. MDR1 expression was present as brown-yellow particles in the plasma membrane and cytoplasm, and GSTP was observed in the nucleus and cytoplasm. IHC revealed that GSTP and MDR1 expression were significantly higher in the 5-Fu-based MTD groups compared with those of the LDM, and control group (Figs. 1 and 2). In terms of GSTP, the mean density of staining positive in the 5-Fu MTD, LDM and control groups was $0.120 \pm 0.01,0.076 \pm 0.001$ and $0.06 \pm 0.001$, respectively, and the 5-Fu LDM group was significantly lower than the 5-Fu MTD dose group $(\mathrm{P}<0.001)$ and slightly higher compared with the control group $(\mathrm{P}<0.05$; Fig. $1 \mathrm{~B})$. The mean density of staining positive in capecitabine MTD, capecitabine LDM and the control groups was $0.134 \pm 0.01$, $0.109 \pm 0.002$ and $0.06 \pm 0.001$, respectively. The mean density in the capecitabine LDM group was significantly lower compared with the capecitabine MTD group $(\mathrm{P}<0.01)$ and higher than the control group $(\mathrm{P}<0.001$; Fig. 1B). In terms of MDR1, the mean density of positive staining in the 5-Fu MTD, 5-Fu LDM and the control groups was $0.058 \pm 0.001$, $0.028 \pm 0.001$ and $0.02 \pm 0.001$, respectively. The 5-Fu LDM group was lower than the 5-Fu MTD group $(\mathrm{P}<0.0001)$, but higher compared with control group ( $\mathrm{P}<0.001$; Fig. $2 \mathrm{~B})$. The mean density of positive staining in the capecitabine MTD, capecitabine LDM and the control groups was $0.052 \pm 0.001,0.040 \pm 0.001$, and $0.02 \pm 0.001$, respectively. The mean density in the capecitabine LDM group was significantly lower than the capecitabine MTD group $(\mathrm{P}<0.0001)$ and higher compared with the control group $(\mathrm{P}<0.0001$; Fig. 2B). Taken together, these observations advocated that the MTD group of capecitabine and 5-Fu may increase the risk of drug resistance when compared with the LDM groups.

Tumor response to treatment is associated with increased frequency of CAFs. Previous studies have demonstrated that stromal compartments are changed by cytotoxic therapies $(14,15)$, indicating microenvironment-associated drug resistance. Therefore, the stromal response in the MTD and LDM groups treated with $5-\mathrm{Fu}$ or capecitabine was examined. CAFs were investigated on the basis of their proposed roles in supporting drug-resistance (16). As the characteristics of CAFs are rather distinctive in different tumor types and stages, without homogeneity, in order to compare the CAF evolution in GC following different patterns of chemotherapies, matched samples from identical xenografts of the control, MTD, and LDM groups were stained for the CAF marker $\alpha$-SMA to reflect the difference (Fig. 3A). The mean density of $\alpha$-SMA in the 5-Fu MTD, 5-Fu LDM, capecitabine MTD, capecitabine LDM and control groups was $0.0374 \pm 0.0015,0.017 \pm 0.001$, $0.0144 \pm 0.0002,0.0122 \pm 0.0002$ and $0.0098 \pm 0.0013$, respectively. IHC demonstrated that $\alpha$-SMA increased following chemotherapy, and the expression in the 5-Fu MTD group was significantly higher compared with that of the 5-Fu LDM group $(\mathrm{P}<0.0001$; Fig. 3B). In addition, the expression of $\alpha$-SMA in the capecitabine MTD group was significantly higher compared with that of the capecitabine LDM group $(\mathrm{P}<0.05$; Fig. 3B). These results indicated that CAFs are enriched during post-therapy tumor growth, particularly following conventional dose traditional chemotherapy.
Effect of different chemotherapy patterns on the expression of $C D 34$ and VEGF. Matched samples from identical xenografts of the control, MTD and LDM groups were stained for CD34, and VEGF (Figs. 4 and 5). The IHC results revealed that the mean density of CD34 in the control group was $0.044 \pm 0.004$, while the mean densities of CD34 in 5-Fu conventional dose and metronomic groups were $0.050 \pm 0.014$, and $0.0216 \pm 0.0009$, respectively. The mean densities of CD34 in the capecitabine conventional dose and metronomic groups were $0.051 \pm 0.004$, and $0.034 \pm 0.003$, respectively. This indicates that $5-\mathrm{Fu}$ and capecitabine conventional dose traditional chemotherapy have no significant effect on the microvascular density (MVD) in GC xenografts, but 5-Fu and capecitabine low dose metronomic chemotherapy significantly decreased the MVD $(\mathrm{P}<0.05$ and $\mathrm{P}<0.01$, respectively; Fig. 4B). These results correspond with those of our previous study revealing that it is 5-FU and capecitabine metronomic chemotherapy rather than 5-FU and capecitabine traditional chemotherapy that decrease the MVD in the GC xenografts (9). The mean densities of VEGF in the 5-Fu MTD, 5-Fu LDM, capecitabine MTD, capecitabine LDM and control groups were 0.016 $\pm 0.001,0.0066 \pm 0.0001$, $0.063 \pm 0.001,0.012 \pm 0.001$, and $0.063 \pm 0.001$, respectively (Fig. 5). The results of IHC demonstrated that, although the level of VEGF in the capecitabine MTD group was similar to those of the control group ( $\mathrm{P}>0.05)$, the VEGF expression was significantly decreased following 5-Fu and capecitabine LDM (both $\mathrm{P}<0.0001$; Fig. 5B).

\section{Discussion}

5-Fu-based chemotherapy served as the first-line treatment of GC (17). Capecitabine is a precursor of 5-Fu and exhibits antitumor effects via conversion by the thymidine phosphorylase enzyme in cancer cells (8). To be an oral cytotoxic agent, capecitabine has significant merits compared with intravenous drugs with regards to being an appropriate choice for metronomic chemotherapy (9). The characteristics of better efficacy, low toxicity and good compliance, which have been confirmed, made metronomic chemotherapy a novel trend in tumor chemotherapy $(8,9)$.

In the present study, 5-Fu-based metronomic chemotherapy significantly reduced the expression level of GSTP and MDR1 compared with those of conventional dose chemotherapy. A previous study has demonstrated that multidrug resistance (MDR) is the main cause for the failure of chemotherapy, particularly in GC, and the occurrence of MDR proceeds through an increased expression level of P-glycoprotein and a decreased level of topoisomerase II (18). MDR1 protein, also termed P-glycoprotein, may reduce the intracellular concentration of chemotherapeutic drugs via inducing the efflux of anticancer drug, and GSTP may protect cells against toxic electrophiles and oxidative stress products, both of which are classical MDR pathways leading to drug resistance (19). Additionally, GSTP-positive GCs are resistant to 5-Fu (20), which corresponds to the present study findings whereby 5-Fu-based conventional chemotherapy group acquired drug-resistance.

To the best of our knowledge, no studies have previously compared the drug resistance abilities between different chemotherapeutic routes. It was found that conventional 
A

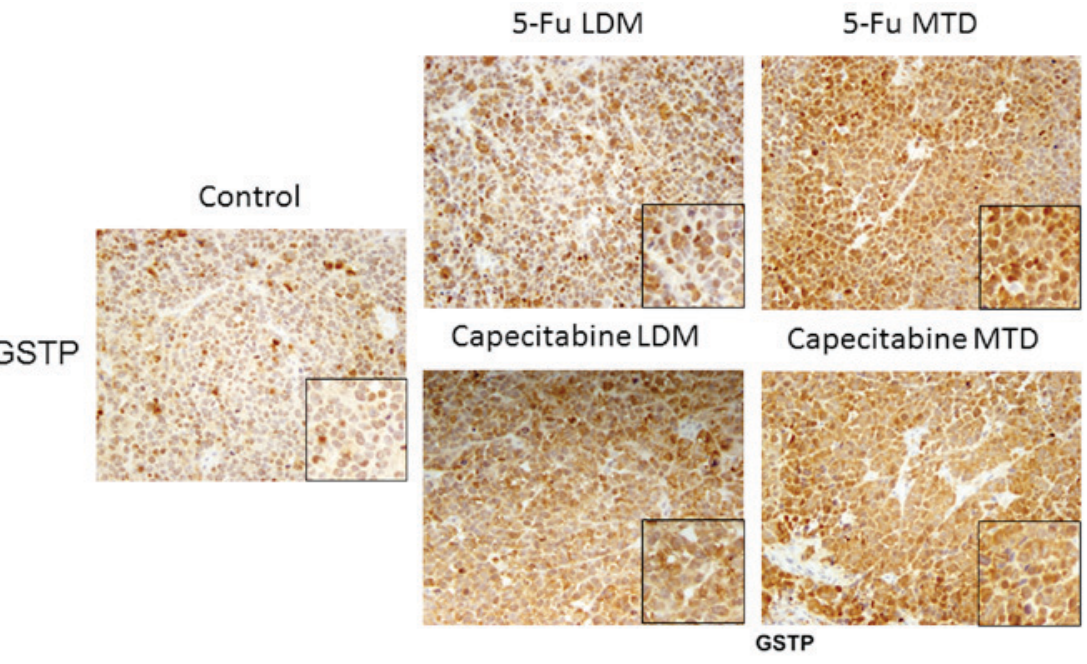

B

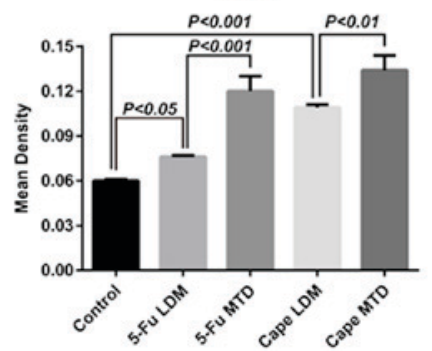

Figure 1. GSTP expression following different chemotherapy regimens of 5-Fu and capecitabine. (A) Immunohistochemical staining for the 5-Fu MTD group, the 5-Fu LDM group, the capecitabine MTD group, the capecitabine LDM group and the control group. (B) Quantification of GSTP expression in the 5-Fu and capecitabine groups. Magnification, x200. 5-Fu, 5-fluorouracil; LDM, low-dose metronomic; MTD, maximum tolerated dose; GSTP, glutathione transferase Pi.

A

B

\section{5-Fu LDM}

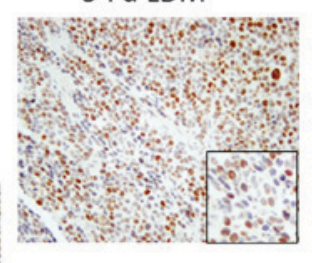

\section{Capecitabine LDM}

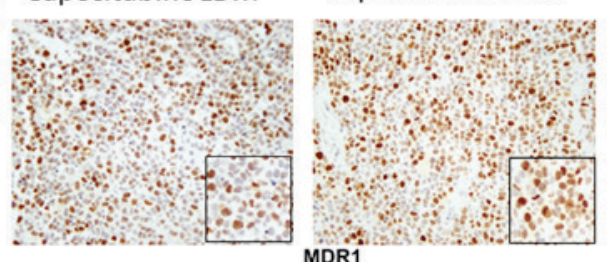

MDR1

5-Fu MTD

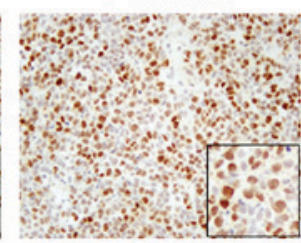

Capecitabine MTD

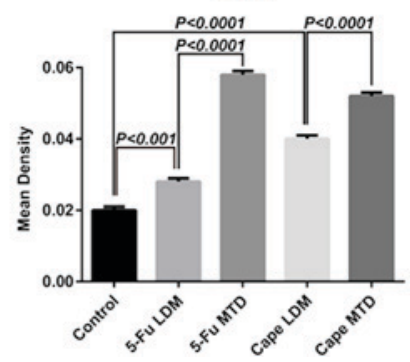

Figure 2. MDR1 expression following different chemotherapy regimens of 5-Fu and capecitabine. (A) Immunohistochemical staining for the 5-Fu MTD group, the 5-Fu LDM group, the capecitabine MTD group, the capecitabine LDM group and the control group. (B) Quantification of MDR1 expression in the 5-Fu and capecitabine groups. Magnification, x200. 5-Fu, 5-fluorouracil; LDM, low-dose metronomic; MTD, maximum tolerated dose; MDR1, multidrug resistance protein 1.

chemotherapy with 5-Fu or capecitabine may increase the risk of drug resistance compared with a metronomic approach.
The increased expression of CAFs in the tissue of patients receiving conventional therapy suggests the possibility of 
A

$\alpha-\mathrm{SMA}$

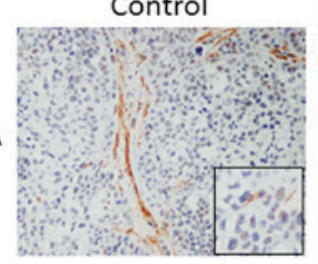

B
5-Fu LDM

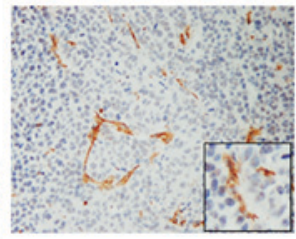

Capecitabine LDM

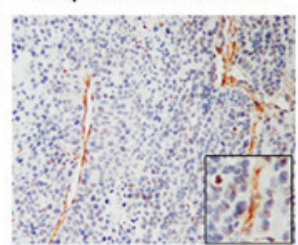

5-Fu MTD

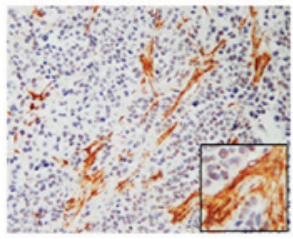

Capecitabine MTD

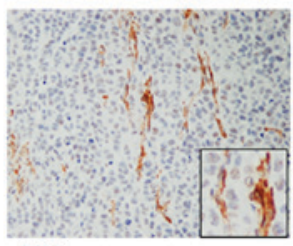

a-SMA

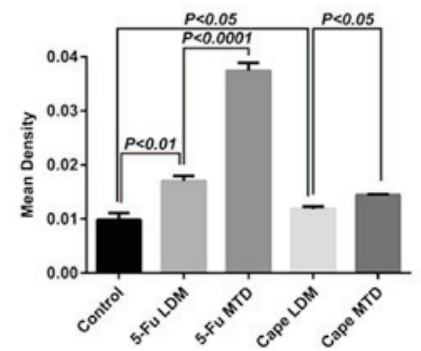

Figure 3.Cancer-associated fibroblasts marker $\alpha$-SMA expression following different chemotherapy regimens of 5-Fu and capecitabine.(A) Immunohistochemical staining for the 5-Fu MTD group, the 5-Fu LDM group, the capecitabine MTD group, the capecitabine LDM group and the control group. (B) Quantification of $\alpha$-SMA expression in the 5-Fu and capecitabine groups. Magnification, x200. 5-Fu, 5-fluorouracil; LDM, low-dose metronomic; MTD, maximum tolerated dose; $\alpha$-SMA, $\alpha$-smooth muscle actin.

A

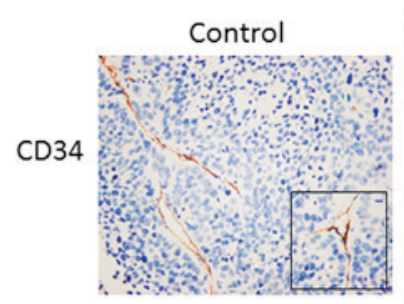

B
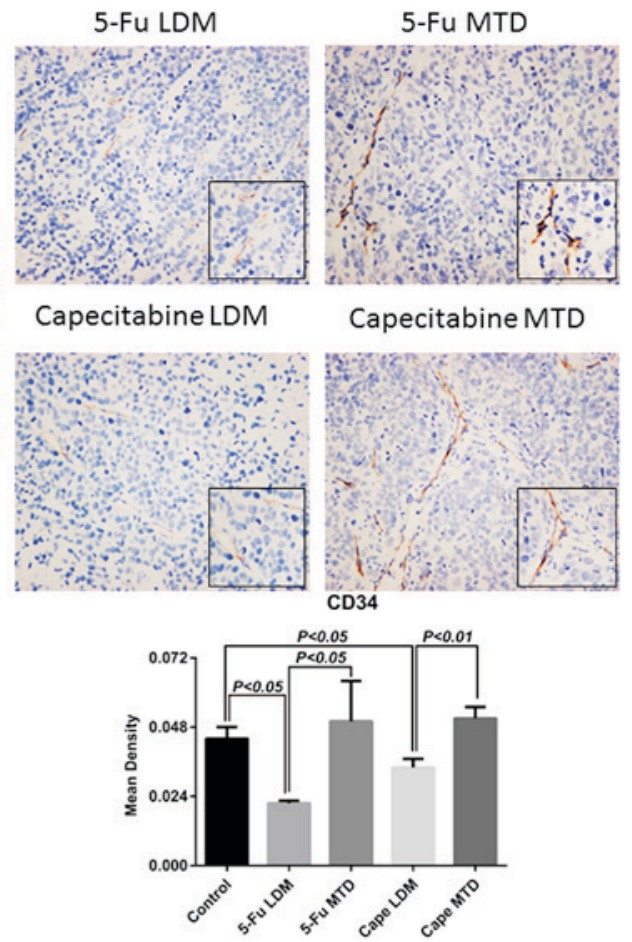

Figure 4. CD34 expression following different chemotherapy regimens of 5-Fu and capecitabine. (A) Immunohistochemical staining for the 5-Fu MTD group, the 5-Fu LDM group, the capecitabine MTD group, the capecitabine LDM group and the control group. (B) Quantification of CD34 expression in the 5-Fu and capecitabine groups. Magnification, x200. 5-Fu, 5-fluorouracil; LDM, low-dose metronomic; MTD, maximum tolerated dose; CD34, cluster of differentiation 34.

drug resistance (16). Thus, it was speculated that CAFs may rebuild the vessel in the microenvironment via the production of VEGF, leading to drug resistance following chemotherapy.
The results in mice have essential clinical implications, which may aid in explaining why a number of patients receiving metronomic or maintenance chemotherapy continue to have 
A

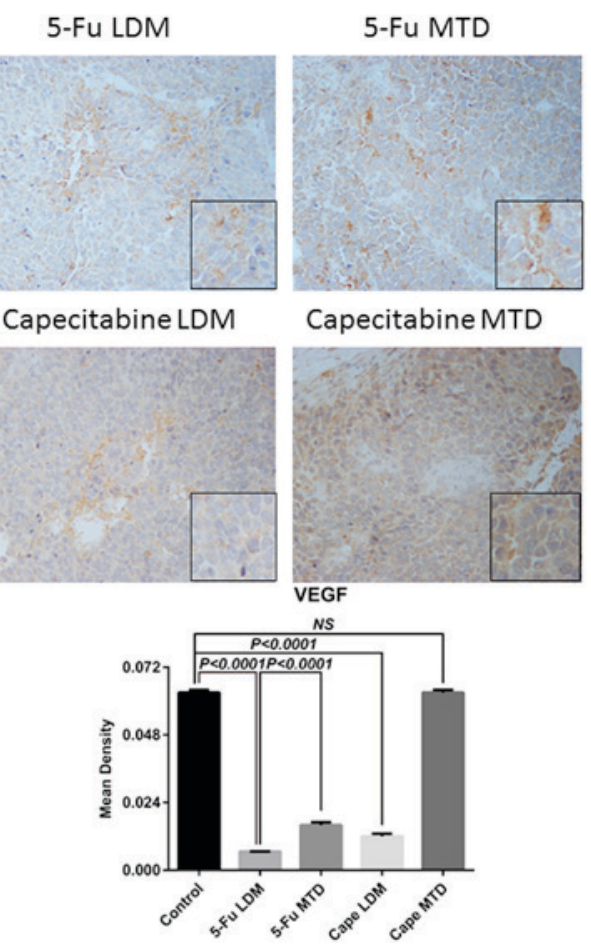

Figure 5. VEGF expression following different chemotherapy regimens of 5-Fu and capecitabine. (A) Immunohistochemical staining for the 5-Fu MTD group, the 5-Fu LDM group, the capecitabine MTD group, the capecitabine LDM group and the control group. (B) Quantification of VEGF expression in the 5-Fu and capecitabine groups. Magnification, x200. 5-Fu, 5-fluorouracil; LDM, low-dose metronomic; MTD, maximum tolerated dose; VEGF, vascular endothelial growth factor.

stable disease exceeding the expected duration for cancer cells to acquire chemoresistance. Studies associated with metronomic chemotherapy mainly focus on anti-angiogenic functions without consideration of the interaction with the microenvironment; however, the change in the tumor microenvironment caused by metronomic chemotherapy may affect the chemoresistance (7-9). The study of metronomic chemotherapy to reduce drug resistance may be of use to improve patient outcomes in clinical practice.

As demonstrated in a previous study (10), the anti-angiogenetic characteristic of cyclophosphamide has been demonstrated through increasing the apoptosis of tumor cells and maintaining the cytotoxic pressure on the vascular endothelial cells within the tumor bed, overall leading to no drug resistance being acquired. These results were not achieved using the conventional schedule, whereby mice harboring tumors developed acquired drug resistance (10). The anti-angiogenetic schedule used by Browder et al (10), is similar to the metronomic chemotherapy in current use, as is the metronomic administration pattern of 5-Fu or capecitabine. All the methods utilize the drugs in innovative ways which are able to have an improved effect over the traditional pattern.

The significant increase in the amount of CAFs following chemotherapy indicated that chemotherapy may induce the remodeling of the tumor microenvironment as well, and CAFs may offer microenvironmental cues instructing tumor drug resistance $(14,21)$. In vitro assays have also demonstrated that CAFs induce resistance to chemotherapy via secreting cytokines $(16,22,23)$. Besides chemotherapy agents, inhibiting CAFs may also enhance the effects of bevacizumab (rhuMab VEGF, Avastin) even in bevacizumab-resistant GC cells (24), which is similar to the selective susceptibility of $\alpha$-SMA-deficient vessels to bevacizumab (25). CAFs are the primarily source of VEGF, cancer epithelial cells are able to produce VEGF and the level of VEGF is increased through the cancer-stromal interaction (26). VEGFs and their receptors have been revealed to modulate vascular permeability activity, leading to enhanced interstitial fluid pressure in the tumor stroma, which is associated with chemotherapeutic resistance (25).

The participation of CAFs in tumor progression and metastasis is well established, particularly in GC $(27,28)$, therefore, anti-CAF therapy may have a triple effect through its anti-angiogenesis potency, antitumor qualities, and the ability to increase chemotherapeutic drugs being absorbed by the tumor (26). Similar to the oral anti-fungal agent itraconazole, which is able to suppress the angiogenetic factors secreted from CAFs, a synergic effect was demonstrated with pemetrexed in a second-line therapy trial for lung cancer $(24,29)$.

In conclusion, the present study demonstrated that low dose metronomic chemotherapy was able to significantly reduce the risk of acquired chemoresistance compared with the normal dose conventional chemotherapy, and the difference in the level of CAFs following both chemotherapy patterns confirmed the diversity of drug-resistance. Furthermore, the downregulation of VEGF expression may not only reflect the anti-angiogenesis effect of metronomic chemotherapy, but also corresponds with the reduced number of CAFs that occur, which may contribute to the development of chemoresistance. To better understand the crosstalk between metronomic chemotherapy and the tumor microenvironment, including CAFs, mechanisms that lead 
to chemoresistance and molecules secreted by CAFs require further characterization.

\section{Acknowledgements}

The study was supported by the National Science Foundation of China (grant nos. 81672327, 81372645 and 81502013), The Program of Shanghai Academic/Technology Research Leader (grant no. 17XD1402600), The Shanghai Municipal Education Commission-Gaofeng Clinical Medicine Grant Support (grant no. 20161410), The FONG SHU FOOK TONG Foundation and National Key Clinical Discipline (Oncology) and the innovation foundation of translational medicine of Shanghai Jiao Tong University School of Medicine (grant no. 15ZH3001), The Program for Outstanding Medical Academic Leader and Shanghai Municipal Commission of Health and Family Planning (grant no. 20154Y496) and SCORE Foundation (grant no. Y-MX2015-078).

\section{References}

1. Torre LA, Bray F, Siegel RL, Ferlay J, Lortet-Tieulent J and Jemal A: Global cancer statistics, 2012. CA Cancer J Clin 65 87-108, 2015.

2. Fujitani K: Overview of adjuvant and neoadjuvant therapy for resectable gastric cancer in the East. Dig Surg 30: 119-129, 2013

3. Bang YJ, Kim YW, Yang HK, Chung HC, Park YK, Lee KH, Lee KW, Kim YH, Noh SI, Cho JY, et al: Adjuvant capecitabine and oxaliplatin for gastric cancer after D2 gastrectomy (CLASSIC): A phase 3 open-label, randomised controlled trial Lancet 379: 315-321, 2012

4. Nakajima T, Kinoshita T, Nashimoto A, Sairenji M, Yamaguchi T, Sakamoto J, Fujiya T, Inada T, Sasako M and Ohashi Y; National Surgical Adjuvant Study of Gastric Cancer Group: Randomized controlled trial of adjuvant uracil-tegafur versus surgery alone for serosa-negative, locally advanced gastric cancer. Br J Surg 94: 1468-1476, 2007

5. Sasako M, Sakuramoto S, Katai H, Kinoshita T, Furukawa H, Yamaguchi T, Nashimoto A, Fujii M, Nakajima T and Ohashi Y: Five-year outcomes of a randomized phase III trial comparing adjuvant chemotherapy with S-1 versus surgery alone in stage II or III gastric cancer. J Clin Oncol 29: 4387-4393, 2011.

6. Banys-Paluchowski M, Schütz F, Ruckhäberle E, Krawczyk N and Fehm T: Metronomic chemotherapy for metastatic breast cancer - a systematic review of the literature. Geburtshilfe Frauenheilkd 76: 525-534, 2016.

7. Pasquier E, Kavallaris M and André N: Metronomic chemotherapy: New rationale for new directions. Nat Rev Clin Oncol 7: 455-465, 2010.

8. Shi H, Jiang J, Ji J, Shi M, Cai Q, Chen X, Yu Y, Liu B, Zhu Z and Zhang J: Anti-angiogenesis participates in antitumor effects of metronomic capecitabine on colon cancer. Cancer Lett 349: 128-135, 2014.

9. Yuan F, Shi H, Ji J, Cai Q, Chen X, Yu Y, Liu B, Zhu Z and Zhang J: Capecitabine metronomic chemotherapy inhibits the proliferation of gastric cancer cells through anti-angiogenesis. Oncol Rep 33: 1753-1762, 2015.

10. Browder T, Butterfield CE, Kräling BM, Shi B, Marshall B, O'Reilly MS and Folkman J: Antiangiogenic scheduling of chemotherapy improves efficacy against experimental drug-resistant cancer. Cancer Res 60: 1878-1886, 2000.

11. Wang W, McLeod HL, Cassidy J and Collie-Duguid ES: Mechanisms of acquired chemoresistance to 5-fluorouracil and tomudex: Thymidylate synthase dependent and independent networks. Cancer Chemother Pharmacol 59: 839-845, 2007.
12. Li J, Guan J, Long X, Wang Y and Xiang X: mir-1-mediated paracrine effect of cancer-associated fibroblasts on lung cancer cell proliferation and chemoresistance. Oncol Rep 35: 3523-3531, 2016.

13. Shintani Y, Fujiwara A, Kimura T, Kawamura T, Funaki S, Minami M and Okumura M: IL-6 secreted from cancer associated fibroblasts mediates chemoresistance in NSCLC by increasing epithelial-mesenchymal transition signaling. J Thorac Oncol 11: 1482-1492, 2016

14. Lotti F, Jarrar AM, Pai RK, Hitomi M, Lathia J, Mace A, Gantt GA Jr, Sukhdeo K, DeVecchio J, Vasanji A, et al: Chemotherapy activates cancer-associated fibroblasts to maintain colorectal cancer-initiating cells by IL-17A. J Exp Med 210: 2851-2872, 2013.

15. Tanaka K, Miyata H, Sugimura K, Fukuda S, Kanemura T, Yamashita K, Miyazaki Y, Takahashi T, Kurokawa Y, Yamasaki M, et al: miR-27 is associated with chemoresistance in esophageal cancer through transformation of normal fibroblasts to cancer-associated fibroblasts. Carcinogenesis 36: 894-903, 2015.

16. Amornsupak K, Insawang $\mathrm{T}$, Thuwajit $\mathrm{P}, \mathrm{O}-\mathrm{Ch}$ aroenrat $\mathrm{P}$, Eccles SA and Thuwajit C: Cancer-associated fibroblasts induce high mobility group box 1 and contribute to resistance to doxorubicin in breast cancer cells. BMC Cancer 14: 955, 2014.

17. Shen L, Shan YS, Hu HM, Price TJ, Sirohi B, Yeh KH, Yang YH, Sano T, Yang HK, Zhang X, et al: Management of gastric cancer in Asia: Resource-stratified guidelines. Lancet Oncol 14: e535-e547, 2013.

18. Zhao W, Chen R, Zhao M, Li L, Fan L and Che XM: High glucose promotes gastric cancer chemoresistance in vivo and in vitro. Mol Med Rep 12: 843-850, 2015.

19. Longley DB and Johnston PG: Molecular mechanisms of drug resistance. J Pathol 205: 275-292, 2005.

20. Geng M, Wang L, Chen X, Cao R and Li P: The association between chemosensitivity and Pgp, GST- $\pi$ and Topo II expression in gastric cancer. Diagn Pathol 8: 198, 2013.

21. Verset L, Tommelein J, Moles Lopez X, Decaestecker C, Boterberg T, De Vlieghere E, Salmon I, Mareel M, Bracke M, De Wever O and Demetter P: Impact of neoadjuvant therapy on cancer-associated fibroblasts in rectal cancer. Radiother Oncol 116: 449-454, 2015.

22. Yan H, Guo BY and Zhang S: Cancer-associated fibroblasts attenuate Cisplatin-induced apoptosis in ovarian cancer cells by promoting STAT3 signaling. Biochem Biophys Res Commun 470: 947-954, 2016.

23. Steinbichler TB, Metzler V, Pritz C, Riechelmann H and Dudas J: Tumor-associated fibroblast-conditioned medium induces CDDP resistance in HNSCC cells. Oncotarget 7: 2508-2518, 2016.

24. Hara M, Nagasaki T, Shiga K and Takeyama H: Suppression of cancer-associated fibroblasts and endothelial cells by itraconazole in bevacizumab-resistant gastrointestinal cancer. Anticancer Res 36: 169-177, 2016.

25. Salnikov AV, Heldin NE, Stuhr LB, Wiig H, Gerber H, Reed RK and Rubin K: Inhibition of carcinoma cell-derived VEGF reduces inflammatory characteristics in xenograft carcinoma. Int J Cancer 119: 2795-2802, 2006.

26. Zhang $\mathrm{J}$ and Liu J: Tumor stroma as targets for cancer therapy. Pharmacol Ther 137: 200-215, 2013.

27. Franco OE, Shaw AK, Strand DW and Hayward SW: Cancer associated fibroblasts in cancer pathogenesis. Semin Cell Dev Biol 21: 33-39, 2010

28. Yan Y, Wang LF and Wang RF: Role of cancer-associated fibroblasts in invasion and metastasis of gastric cancer. World J Gastroenterol 21: 9717-9726, 2015.

29. Rudin CM, Brahmer JR, Juergens RA, Hann CL, Ettinger DS, Sebree R, Smith R, Aftab BT, Huang P and Liu JO: Phase 2 study of pemetrexed and itraconazole as second-line therapy for metastatic nonsquamous non-small-cell lung cancer. J Thorac Oncol 8: 619-623, 2013. 